\title{
Editors' Report for 2019
}

Dan Bogart is now in his second year editing the Eurasian submissions to the JOURNAL, and Bill Collins is in his fourth and final year editing the non-Eurasian submissions. Eric Hilt continues his work as book review editor for the Americas, and Aldo Musacchio continues his work as book review editor for the other parts of the world. The JOURNAL is fortunate in having Lily Welch continue as the JourNAL's Editorial Assistant and Sally Sztrecska as its Production Editor.

The Journal has benefited greatly from the expert guidance and advice of our Editorial Board and more than 200 referees who contributed their time and expertise to maintaining and enhancing the JouRnAL's quality. Editorial Board members typically serve four-year terms. Those departing from the Board this year are Guido Alfani, Richard Hornbeck, Morgan Kelly, Noel Maurer, Petra Moser, Aldo Musacchio, Kim Oosterlinck, and Warren Weber. Carola Frydman and Marianne Wanamaker have left the Board to become editors of Explorations in Economic History. Kerstin Enflo has left the Board to become an editor of the European Review of Economic History. New board members include Leticia Arroyo Abad, Eric Hornung, Carl Kitchens, Jared Rubin, Mohamed Saleh, and Laura Salisbury. We also recognize and appreciate numerous scholars' contributions to the JOURNAL's book reviews. The editorial office is grateful to all who have worked on behalf of the JOURNAL over this past year.

The number of new submissions to the JouRnAL in recent years is shown in Table 1. Over the last several years, the average number of submissions per year is approximately 160 , but in the past year there was a noticeable jump in the number of submissions. Time will tell whether this is an aberration or a new level for the inflow of submissions. The publication ratio displayed in Table 1 is the number of refereed papers and notes published in the current year divided by the number of new manuscripts submitted in the previous year. In 2018-2019, the publication ratio was $0.18(28 / 155)$, which is in line with the recent past. The JouRnal published 30 articles in total in 2018-2019, including Cormac Ó Gráda's presidential address and Kevin O’Rourke’s "reviews and reflections" essay.

Table 2 reports the response-time statistics for the corresponding sample. To place the numbers into context, our goal is to have a decision back to the author within 90 days. This past year's performance has been somewhat faster than previous years for new submissions, with a median response time of just under 60 days. Note that this pertains to submissions received from July 2018 to June 2019, some of which may not have received decisions at the time of this report's writing. The median response time for all submissions (included revised manuscripts) is slightly lower. Unfortunately, a small number of papers have taken longer to review. We apologize to authors of manuscripts that experienced substantial delays. We continuously work to reduce the time for this small number of manuscripts, taking into consideration that we are, of course, concerned about the quality of the response, not only its speed.

The distributions of submissions are documented in subsequent tables. We show the data for eras in Table 3 and for topics in Table 4. The automated part of the paper submission system stopped collecting information on regions for a period of time during 2015 and 2016. This means that we do not have consistent comparisons over all years in the table. What is currently available is shown in Table 5.

The JOURNAL's coverage remains broad. Table 3 shows that the distribution of submissions over eras has been fairly steady in recent years, with the nineteenth and twentieth 
centuries accounting for the bulk of submissions. Table 4 shows that submissions in political economy, labor, and growth continue to be particularly strong. Nonetheless, the top-three topic areas together account for less than half of all submissions, reflecting broadly dispersed interests within the field. Table 5 shows that submissions on Western Europe and the United States are most common. In the past year, there was a dip in the share of submissions on Asia and a slight rise in the share of submissions on Africa, Eastern Europe/Russia, and Latin America.

Book reviews are an important component of the JOURNAL, and we sincerely thank the many reviewers. We also remind our membership to have their press send a copy of any new books to the relevant book review editor. In recent years, the JouRnAL has also sought occasional "reviews and reflections" synthetic articles, extended book reviews, and roundtable book reviews when appropriate. For instance, in June 2019, the JoURNAL published "Economic History and Contemporary Challenges to Globalization," a "reviews and reflections" article by Kevin O'Rourke.

Readers will recall that at the 2013 meetings, the EHA board of trustees approved a policy to make publication conditional on archiving the data sufficient to replicate the results in the accepted articles. The authors of all new submissions from 1 January 2016 onward that were accepted for publication have been required to post data and replication files to maintain and advance the JOURNAL's efforts to ensure transparency and scholarly integrity. A branded page can be found with openICPSR, and depositors can submit files into openICPSR per the instructions available at: http://www.icpsr.umich. edu/files/openICPSR/JEH-deposit-instructions.pdf. The editorial office and ICPSR continue to work to enhance the interface.

TABLE 1

SUBMISSION AND PUBLICATION RATES

\begin{tabular}{lccc}
\hline \hline & $\begin{array}{c}\text { Published September } \\
\text { to June issues }\end{array}$ & $\begin{array}{c}\text { New Submissions, } \\
\text { "New" Method }\end{array}$ & $\begin{array}{c}\text { Publication Ratio } \\
\text { Pt/St-1 }\end{array}$ \\
\hline $2011-2012$ & 32 & 152 & 0.21 \\
$2012-2013$ & 31 & 143 & 0.20 \\
$2013-2014$ & 32 & 158 & 0.22 \\
$2014-2015$ & 32 & 177 & 0.20 \\
$2015-2016$ & 31 & 170 & 0.18 \\
$2016-2017$ & 29 & 162 & 0.17 \\
$2017-2018$ & 29 & 155 & 0.18 \\
$2018-2019$ & 28 & 195 & 0.18 \\
\hline
\end{tabular}

The "published" count includes refereed articles and notes. It does not include the presidential address, which is published in June, nor does it include "reviews and reflections" articles. In 2017-2018, the JOURNAL published one "reviews and reflections" article. 
TABLE 2

RESPONSE TIME STATISTICS

\begin{tabular}{|c|c|c|c|c|}
\hline \multirow[b]{2}{*}{ Year } & \multicolumn{2}{|c|}{ Decision Time (in days) } & \multirow[b]{2}{*}{ Mean } & \multirow[b]{2}{*}{ Median } \\
\hline & Minimum & Maximum & & \\
\hline \multicolumn{5}{|c|}{ All submissions } \\
\hline $2012 / 2013$ & 2 & 237 & 80 & 78 \\
\hline $2013 / 2014$ & 4 & 243 & 76 & 75 \\
\hline $2015 / 2015$ & 1 & 235 & 78 & 78 \\
\hline $2015 / 2016$ & 1 & 277 & 60 & 50 \\
\hline $2016 / 2017$ & 1 & 306 & 61 & 60 \\
\hline $2017 / 2018$ & 1 & 227 & 51 & 47 \\
\hline 2018/2019 & 1 & 175 & 48 & 56 \\
\hline \multicolumn{5}{|c|}{ New submissions } \\
\hline $2012 / 2013$ & 2 & 237 & 86 & 85 \\
\hline $2013 / 2014$ & 4 & 243 & 80 & 79 \\
\hline $2014 / 2015$ & 1 & 235 & 80 & 78 \\
\hline $2015 / 2016$ & 1 & 277 & 74 & 74 \\
\hline $2016 / 2017$ & 1 & 306 & 73 & 75 \\
\hline $2017 / 2018$ & 1 & 227 & 64 & 64 \\
\hline $2018 / 2019$ & 1 & 175 & 56 & 59 \\
\hline
\end{tabular}

TABLE 3

DISTRIBUTION OF SUBMISSIONS BY ERA, MULTIPLE SELECTIONS PER SUBMISSION POSSIBLE

\begin{tabular}{lrrrrrr}
\hline \hline & $2013-$ & $2014-$ & $2015-$ & $2016-$ & $2017-$ & $2018-$ \\
Eras & 2014 & 2015 & 2016 & 2017 & 2018 & 2019 \\
\hline Pre-seventeenth century & 22 & 28 & 24 & 15 & 17 & 17 \\
Seventeenth and eighteenth & 39 & 43 & 24 & 27 & 29 & 37 \\
$\quad$ centuries & & & & & & \\
Nineteenth century & 84 & 92 & 56 & 74 & 72 & 83 \\
Twentieth century & 87 & 104 & 78 & 83 & 77 & 100 \\
Twenty-first century & 16 & 12 & 15 & 16 & 10 & 14 \\
Not applicable & 2 & 2 & 3 & 4 & 8 & 5 \\
Total & 250 & 281 & 200 & 219 & 213 & 256 \\
\hline & & Percent & & & & \\
\hline Pre-seventeenth century & 8.8 & 10.0 & 12.0 & 6.8 & 8.0 & 6.6 \\
Seventeenth and eighteenth & 15.6 & 15.3 & 12.0 & 12.3 & 13.6 & 14.5 \\
$\quad$ centuries & & & & & & \\
Nineteenth century & 33.6 & 32.7 & 28.0 & 33.8 & 33.8 & 32.4 \\
Twentieth century & 34.8 & 37.0 & 39.0 & 37.9 & 36.2 & 39.1 \\
Twenty-first century & 6.4 & 4.3 & 7.5 & 7.3 & 4.7 & 5.5 \\
Not applicable & 0.8 & 0.7 & 1.5 & 1.8 & 3.8 & 2.0 \\
\hline
\end{tabular}

Note: This table corrects errors in previous year's report for 2014-2015 pre-seventeenth-century percent entry and for 2015-2016 twentieth-century percent entry. 
TABLE 4

DISTRIBUTION OF SUBMISSIONS BY TOPIC, MULTIPLE SELECTIONS

PER SUBMISSION POSSIBLE

\begin{tabular}{|c|c|c|c|c|c|c|}
\hline Topics & $\begin{array}{l}2013- \\
2014\end{array}$ & $\begin{array}{l}2014- \\
2015\end{array}$ & $\begin{array}{l}2015- \\
2016\end{array}$ & $\begin{array}{l}2016- \\
2017\end{array}$ & $\begin{array}{l}2017- \\
2018\end{array}$ & $\begin{array}{l}2018- \\
2019\end{array}$ \\
\hline Agriculture & 24 & 19 & 8 & 10 & 11 & 18 \\
\hline Demography & 25 & 24 & 25 & 22 & 17 & 17 \\
\hline Growth & 26 & 39 & 36 & 28 & 24 & 30 \\
\hline History of thought & 3 & 4 & 7 & 3 & 5 & 10 \\
\hline Industry & 20 & 19 & 8 & 7 & 13 & 18 \\
\hline International trade, finance & 31 & 20 & 15 & 20 & 14 & 16 \\
\hline Labor & 22 & 23 & 24 & 31 & 31 & 28 \\
\hline Money and macro & 17 & 23 & 16 & 11 & 17 & 13 \\
\hline Other & 22 & 18 & 10 & 12 & 10 & 17 \\
\hline Political economy & 36 & 35 & 31 & 41 & 36 & 46 \\
\hline Private finance, capital markets & 19 & 19 & 12 & 12 & 9 & 17 \\
\hline Public finance & 17 & 17 & 12 & 9 & 18 & 16 \\
\hline Technology & 9 & 13 & 11 & 11 & 0 & 7 \\
\hline Urban and regional & 10 & 10 & 13 & 13 & 16 & 16 \\
\hline Total & 281 & 283 & 228 & 230 & 221 & 269 \\
\hline \multicolumn{7}{|c|}{ Percent } \\
\hline Agriculture & 8.5 & 6.7 & 3.5 & 4.3 & 5.0 & 6.7 \\
\hline Demography & 8.9 & 8.5 & 11.0 & 9.6 & 7.7 & 6.3 \\
\hline Growth & 9.3 & 13.8 & 15.8 & 12.2 & 10.9 & 11.2 \\
\hline History of thought & 1.1 & 1.4 & 3.1 & 1.3 & 2.3 & 3.7 \\
\hline Industry & 7.1 & 6.7 & 3.5 & 3.0 & 5.9 & 6.7 \\
\hline International trade, finance & 11.0 & 7.1 & 6.6 & 8.7 & 6.3 & 5.9 \\
\hline Labor & 7.8 & 8.1 & 10.5 & 13.5 & 14.0 & 10.4 \\
\hline Money and macro & 6.0 & 8.1 & 7.0 & 4.8 & 7.7 & 4.8 \\
\hline Other & 7.8 & 6.4 & 4.4 & 5.2 & 4.5 & 6.3 \\
\hline Political economy & 12.8 & 12.4 & 13.6 & 17.8 & 16.3 & 17.1 \\
\hline Private finance, capital markets & 6.8 & 6.7 & 5.3 & 5.2 & 4.1 & 6.3 \\
\hline Public finance & 6.0 & 6.0 & 5.3 & 3.9 & 8.1 & 5.9 \\
\hline Technology & 3.2 & 4.6 & 4.8 & 4.8 & 0.0 & 2.6 \\
\hline Urban and regional & 3.6 & 3.5 & 5.7 & 5.7 & 7.2 & 5.9 \\
\hline
\end{tabular}

Note: This table corrects errors in previous years' reports for 2014-2015 and 2015-2016 percent entries. 
TABLE 5

DISTRIBUTION OF GEOGRAPHICAL AREA

\begin{tabular}{|c|c|c|c|c|c|c|}
\hline Regions & $\begin{array}{c}2013- \\
2014\end{array}$ & $\begin{array}{c}* 2014- \\
2015\end{array}$ & $\begin{array}{c}* 2015- \\
2016 \\
\end{array}$ & $\begin{array}{c}2016- \\
2017 \\
\end{array}$ & $\begin{array}{c}2017- \\
2018\end{array}$ & $\begin{array}{r}2018 \\
2019 \\
\end{array}$ \\
\hline Africa & 8 & 3 & 2 & 13 & 5 & 16 \\
\hline Asia & 19 & 4 & 6 & 28 & 34 & 28 \\
\hline Australia and New Zealand & 3 & 3 & 0 & 5 & 1 & 2 \\
\hline Eastern Europe/Russia & 15 & 2 & 3 & 13 & 7 & 15 \\
\hline Great Britain & 36 & 10 & 2 & 29 & 18 & 26 \\
\hline Latin America & 19 & 4 & 1 & 6 & 7 & 12 \\
\hline Middle East & 7 & 2 & 3 & 9 & 7 & 5 \\
\hline $\begin{array}{l}\text { Non-Spanish speaking } \\
\text { Caribbean }\end{array}$ & 4 & 0 & 1 & 1 & 3 & 1 \\
\hline United States and Canada & 65 & 16 & 10 & 48 & 43 & 58 \\
\hline Western Europe & 56 & 14 & 6 & 49 & 53 & 70 \\
\hline Not applicable & 3 & 0 & 3 & 2 & 6 & 3 \\
\hline Total & 235 & 58 & 37 & 203 & 184 & 236 \\
\hline \multicolumn{7}{|c|}{ Percent } \\
\hline Africa & 3.4 & 5.2 & 5.4 & 6.4 & 2.7 & 6.8 \\
\hline Asia & 8.1 & 6.9 & 16.0 & 13.8 & 18.5 & 11.9 \\
\hline Australia and New Zealand & 1.3 & 5.2 & 0 & 2.5 & 0.5 & 0.8 \\
\hline Eastern Europe/Russia & 6.4 & 3.4 & 8.1 & 6.4 & 3.8 & 6.4 \\
\hline Great Britain & 15.3 & 17.2 & 5.4 & 14.3 & 9.8 & 11.0 \\
\hline Latin America & 8.1 & 6.9 & 2.7 & 3.0 & 3.8 & 5.1 \\
\hline Middle East & 3.0 & 3.4 & 8.1 & 4.4 & 3.8 & 2.1 \\
\hline $\begin{array}{l}\text { Non-Spanish speaking } \\
\text { Caribbean }\end{array}$ & 1.7 & 0 & 2.7 & 0.5 & 1.6 & 0.4 \\
\hline United States and Canada & 27.7 & 27.6 & 27.0 & 23.6 & 23.4 & 24.6 \\
\hline Western Europe & 23.8 & 24.1 & 16.2 & 24.1 & 28.8 & 29.7 \\
\hline Not applicable & 1.3 & 0 & 8.1 & 1.0 & 3.3 & 1.3 \\
\hline
\end{tabular}

*2014-2015 Region data are from 1 July 2014-14 October 2015. 2015-2016 Region data are from 18 March 2016-30 June 2016.

Referees for 2018 were:

Brian A'Hearn

Olivier Accominotti

Scott Adler

Toke Aidt

Guido Alfani

Bob Allen

Marcella Alsan
Lee J. Alston

Belinda Archibong

Leticia Arroyo Abad

Vellore Arthi

Cihan Artunç

Jeremy Atack

Richard Baker
Gerben Bakker

Charlotte Bartels

Brian Beach

Sascha O. Becker

Thor Berger

Asaf Bernstein

Vincent Bignon 


\begin{tabular}{|c|c|c|}
\hline Matthias Blum & James Feigenbaum & Kris Inwood \\
\hline Gustavo Bobonis & Alan Fernihough & Douglas Irwin \\
\hline Leah Boustan & Daniel Fetter & Murat Iyigun \\
\hline Stephen Broadberry & Price Fishback & David Jacks \\
\hline John Brown & Martin Fiszbein & Matthew Jaremski \\
\hline Gillian Brunet & Marc Flandreau & Taylor Jaworski \\
\hline Liam Brunt & Erin Fletcher & Saumitra Jha \\
\hline Carsten Burhop & Caroline Fohlin & Ruixue Jia \\
\hline Joyce Burnette & Nathan Foley-Fisher & Reka Juhasz \\
\hline Edmund Cannon & Johan Fourie & Brooks Kaiser \\
\hline Ann Carlos & Ewout Frankema & Shawn Kantor \\
\hline Celeste Carruthers & Carola Frydman & Daniel Kaufmann \\
\hline Federica Carugati & Dustin Frye & Ian Keay \\
\hline Benjamin Chabot & Leigh Gardner & Morgan Kelly \\
\hline Christophe Chamley & Thomas Gehrig & Lionel Kesztenbaum \\
\hline Eric Chaney & Oscar Gelderblom & Carl Kitchens \\
\hline Latika Chaudhary & Michela Giorcelli & Alexander Klein \\
\hline David Chilosi & Sun Go & John Komlos \\
\hline Dylan Connor & Regina Grafe & Mark Koyama \\
\hline Gary Cox & Rowena Gray & Sumner La Croix \\
\hline Neil Cummins & Farley Grubb & José Miguel Lana-Berasain \\
\hline Tomas Cvrcek & Timothy Guinnane & Marc Law \\
\hline John Dalton & Bishnupriya Gupta & Sibylle Lehmann-Hasemeyer \\
\hline Aditya Dasgupta & Federico Gutierrez & Frank Lewis \\
\hline Joseph Davis & Michael Haines & Dan Li \\
\hline Charles de Bartolome & Walker Hanlon & Gary Libecap \\
\hline Alan de Bromhead & Leslie Hannah & Peter Lindert \\
\hline Mark Dincecco & Timothy Hatton & Trevon Logan \\
\hline Jordi Domenech & Joshua Hausman & Paul-Vincent Lombardi \\
\hline Mauricio Drelichman & Ron Hayduk & Jason Long \\
\hline Martin Dribe & Thomas Heinrich & Sara Lowes \\
\hline Nicolas Duquette & Morgan Henderson & Debin Ma \\
\hline Andrew Dustan & Carlos Eduardo Hernández & Brian Marein \\
\hline Alan Dye & Alfonso Herranz-Loncán & Robert Margo \\
\hline Shari Eli & Eric Hilt & Catherine Massey \\
\hline Kerstin Enflo & Philip Hoffman & Kate Masur \\
\hline Steven A. Epstein & Richard Hornbeck & Noel Maurer \\
\hline Katherine Eriksson & Erik Hornung & Christopher Meissner \\
\hline José-Antonio Espín-Sánchez & Anton Howes & Keith Meyers \\
\hline Giovanni Federico & Jane Humphries & Melinda Miller \\
\hline
\end{tabular}


Chris Minns

Paul Miranti

Rinchan Mirza

Salvatore Morelli

Petra Moser

Charles Moul

Tomas Murphy

Peter Murrell

Aldo Musacchio

Steven Nafziger

Larry Neal

Tom Nicholas

Greg Niemesh

Sergey Nigai

Pilar Nogues-Marco

Johannes Norling

Alessandro Nuvolari

Cormac Ó Gráda

Sheilagh Ogilvie

Kim Oosterlinck

Nuno Palma

Craig Palsson

Sevket Pamuk

Andrea Papadia

John Parman

Helen Paul

Santiago Pérez

Alexander Persaud

Maarten Prak

Jonathan Pritchett

Sarah Reber

Angela Redish
Claudia Rei

Paul Rhode

Gary Richardson

Albrecht Ritschl

Hugh Rockoff

Jonathan Rose

Joshua Rosenbloom

Jean-Laurent Rosenthal

Elyce J. Rotella

Jared Rubin

Martin Saavedra

Mohamed Saleh

Laura Salisbury

Eric Schneider

Max-Stephan Schulze

Andrew Seltzer

Edson Severnini

Paul Sharp

Allison Shertzer

Katharine Shester

Carol Shiue

Pablo Sierra Silva

Peter Siminski

James Siodla

Carolyn Sissoko

Peter Solar

Rohini Somanathan

Arthur Spirling

Steven Sprick Schuster

Bjarne Strøm

David Stuckler

Nathan Sussman
Richard Sutch

Anand Swamy

Hui Ren Tan

John Tang

Peter Temin

Melissa Thomasson

Werner Troesken

John Turner

Stefano Ugolini

Marlous van Waijenburg

Jan Luiten Van Zanden

Jessica Vechbanyongratana

François Velde

Jordi Vidal-Robert

Tamas Vonyo

Daniel Waldenström

Patrick Wallis

Marianne Wanamaker

Kirsten Wandschneider

Zachary Ward

Warren Weber

Marc Weidenmier

Leonardo Weller

Alex Whalley

Eugene White

Gavin Wright

Yishay Yafeh

Noam Yuchtman

Shuang Zhang

Nicolas Ziebarth

Ariell Zimran 